\title{
Pain Education With Therapeutic Exercise in Chronic Nonspecific Low Back Pain Rehabilitation: A Critically Appraised Topic
}

\author{
Kaitlyn C. Jones, Evelyn C. Tocco, Ashley N. Marshall, \\ Tamara C. Valovich McLeod, and Cailee E. Welch Bacon
}

\begin{abstract}
Clinical Scenario: Low back pain is widely prevalent in the general population as well as in athletes. Therapeutic exercise is a lowrisk and effective treatment option for chronic pain that can be utilized by all rehabilitation clinicians. However, therapeutic exercise alone does not address the psychosocial aspects that are associated with chronic low back pain. Pain education is the umbrella term utilized to encompass any type of education to the patient about their chronic pain. Therapeutic exercise in combination with pain education may allow for more well-rounded and effective treatment for patients with chronic nonspecific low back pain (NS-LBP). Clinical Question: Does pain education combined with therapeutic exercise, compared with therapeutic exercise alone, improve patient pain in adults with chronic NS-LBP over a 2- to 3-month treatment period? Summary of Key Findings: A thorough literature review yielded 8 studies potentially relevant to the clinical question, and 3 studies that met the inclusion criteria were included. The 3 studies included reports that exercise therapy reduced symptoms. Two of the 3 included studies support the claim that exercise therapy reduces the symptoms of chronic NS-LBP when combined with pain education, whereas one study found no difference between pain education with therapeutic exercise. Clinical Bottom Line: There is moderate evidence to support the use of pain education along with therapeutic exercise when attempting to reduce symptoms of pain and disability in patients with chronic NS-LBP. Educational interventions should be created to educate patients about the foundation of pain, and pain education should be implemented as a part of the clinician's strategy for the rehabilitation of patients with chronic NS-LBP. Strength of Recommendation: Grade B evidence exists to support the use of patient education with therapeutic exercise for decreasing pain in patients with chronic NS-LBP.
\end{abstract}

Keywords: chronic pain, patient education, treatment-based classification

\section{Clinical Scenario}

Low back pain (LBP) is widely prevalent in the general population as well as in athletics. Though the prevalence is variable depending on the sport, up to $94 \%$ of the athletic population will have LBP over their lifetime. ${ }^{1}$ Chronic nonspecific LBP (NS-LBP) is defined as idiopathic LBP that lasts for 12 weeks or more. ${ }^{2-4}$ Chronic NS-LBP can have a long and tedious treatment that often is not a straightforward process. Psychosocial factors have been well established to have an association with chronic $\mathrm{LBP}^{5-7}$ as has the advocation for including strategies to address these factors during treatment, even in athletes. ${ }^{8}$ The lack of structural deformity or tissue damage associated with chronic NS-LBP can leave both the patients and the clinicians frustrated with the slow rehabilitation process. Common tools utilized by sports medicine clinicians for treating chronic NS-LBP, such as the treatment-based classification system, do not necessarily translate to chronic pain and notably do not take psychosocial factors into consideration. ${ }^{9}$

Therapeutic exercise is a low-risk and effective treatment option for chronic pain, which can be utilized by all rehabilitation clinicians. ${ }^{10}$ However, as with the treatment-based classification, therapeutic exercise alone does not address the psychosocial aspects that are associated with chronic LBP. Opioid medications have historically been subscribed for chronic pain, ${ }^{11}$ but physicians

Jones, Tocco, Valovich McLeod, and Welch Bacon are with Athletic Training Programs, A.T. Still University, Mesa, AZ, USA. Marshall is with Appalachian State University, Boone, NC, USA. Valovich McLeod and Welch Bacon are also with the School of Osteopathic Medicine in Arizona, A.T. Still University, Mesa, AZ, USA. Welch Bacon (cwelch@atsu.edu) is corresponding author. should prescribe with caution due to the mainstream opioid abuse epidemic. Not only do opioids have a high risk for addiction, but also there is inconclusive evidence that their persistent use is effective for pain management. ${ }^{11}$

Pain education is the umbrella term utilized to encompass any type of education to the patient about their chronic pain. Different studies use different terms, such as Neurophysiological Pain Education, to describe their specific methods of educating the patient. Moseley and Butler's ${ }^{12}$ "Explaining Pain" intervention set out to utilize the biopsychosocial model to treat chronic pain. The educational intervention seeks to educate patients about the biology of pain "to shift one's conceptualization of pain from that of a marker of tissue damage or disease to that of a marker of the perceived need to protect body tissue."12(p808) Pain education differs from cognitive behavioral therapy, another commonly utilized pain management technique, in that cognitive behavioral therapy is psychotherapeutic in nature and is not in the scope of practice of the rehabilitation clinicians. ${ }^{13,14}$ Utilizing pain education alone would encounter similar limitations that utilize solely therapeutic exercise does-it would make the biopsychosocial model of patient-centered care incomplete as they focus individually on different aspects of chronic pain care. Therapeutic exercise in combination with pain education may allow for more wellrounded and effective treatment for patients with chronic NS-LBP.

\section{Clinical Question}

Does pain education combined with therapeutic exercise, compared with therapeutic exercise alone, improve patient pain in adults with chronic NS-LBP over a 2- to 3-month treatment period? 


\section{Summary of Search, Best Evidence Appraised, and Key Findings}

- The literature was searched for studies that investigated the effects of exercise therapy and pain education in patients with chronic NS-LBP.

- The literature search returned 8 possible studies ( 3 randomized controlled trials, 2 meta-analysis, 1 observational study, 1 validation study, and 1 case comparison study) related to the clinical question. Three studies met the inclusion criteria and were included. ${ }^{2-4}$

- The 3 studies $^{2-4}$ included reports that exercise therapy reduced symptoms.

- Two of the 3 included studies ${ }^{2-4}$ support the claim that exercise therapy reduces the symptoms of chronic NS-LBP when combined with pain education.

- One study ${ }^{2}$ found no difference between pain education with therapeutic exercise and only therapeutic exercise through patient-reported pain.

\section{Clinical Bottom Line}

There is moderate evidence to support the use of pain education along with therapeutic exercise when attempting to reduce symptoms of pain and disability in patients with chronic NS-LBP. ${ }^{2-4} \mathrm{~A}$ patient's perception of pain can be improved based on specific management and control. Therefore, educational interventions should be created to educate patients about the foundation of pain and to create an image of pain that embodies the need for the body to heal. Pain education should be implemented as a part of the clinician's strategy for the rehabilitation of patients with chronic NS-LBP.

\section{Strength of Recommendation}

Grade B evidence exists to support the use of patient education with therapeutic exercise for decreasing pain in patients with chronic NS-LBP.

\section{Search Strategy}

\section{Terms Used to Guide Search Strategy}

- Patient/Client Group: adults with chronic NS-LBP

- Intervention (or Assessment): pain education with therapeutic exercise

- Comparison: therapeutic exercise alone

- Outcome(s): pain rating scale

\section{Sources of Evidence Searched}

- PubMed

- The Cochrane Library
- MEDLINE

- CINAHL

- EBSCO

- Additional resources obtained via review of reference lists and hand search

\section{Inclusion and Exclusion Criteria}

\section{Inclusion Criteria}

- Studies that used either the visual analog scale or numerical pain rating scale

- Limited to pain education and therapeutic exercise versus therapeutic exercise

- Limited to studies available in English

- Limited to studies conducted in the last 10 years (2010-2019)

\section{Exclusion Criteria}

- Studies in which the participants were prescribed cognitive behavioral therapy

- Studies that included participants with acute LBP

- Studies that included participants with diagnosed structural abnormalities associated with their LBP

- Studies that included participants with chronic/systemic illnesses

\section{Results of Search}

Three relevant studies ${ }^{2-4}$ were located and categorized as shown in Table 1 (based on Levels of Evidence, Centre for Evidence-Based Medicine, 2011).

\section{Best Evidence}

The studies ${ }^{2-4}$ in Table 2 were identified as best evidence and selected the inclusion criteria for the critically appraised topic.

\section{Implications for Practice, Education, and Future Research}

Two of the 3 studies $^{2-4}$ appraised for this topic found that patients who had pain education along with therapeutic exercise experience a greater improvement in self-reported pain rating scale compared with patients who only had therapeutic exercise. Sports medicine and rehabilitation clinicians should consider utilizing pain education in the management of patients with chronic NS-LBP. Methods such as Moseley and Butler's "Explain Pain" 12 can aid in reducing patient-reported pain intensity when combined with a therapeutic exercise program. In addition, improvements in functional

Table 1 Summary of Study Designs of Articles Retrieved

\begin{tabular}{|c|c|c|c|}
\hline Level of evidence & Study design & Number located & Author (year) \\
\hline 2 & Single-blind randomized controlled trial & 3 & $\begin{array}{l}\text { Dufour et al }(2010)^{2} \\
\text { Bodes Pardo et al }(2018)^{3} \\
\text { Pires et al }(2015)^{4}\end{array}$ \\
\hline
\end{tabular}


Table 2 Characteristics of Included Studies

\begin{tabular}{ll}
\hline & Dufour et al (2010) \\
\hline Study design & Randomized controlled trial \\
Participants & 286 patients with chronic NS-LBP between \\
& January 2002 and November 2003 were \\
referred by general practitioners and rheu- & \\
matologists in Copenhagen. & \\
& \\
Biopsychosocial education group \\
(n= 129$):$ \\
Age: \\
$\bullet 18<$ age $\leq 40(46.6 \%)$ \\
$\bullet 40<$ age $\leq 60(53.4 \%)$ \\
Gender: \\
$\bullet$ Male $(43.4 \%)$ \\
$\bullet$ Female $(56.6 \%)$ \\
VAS pain average $(0-100 \mathrm{~mm}): 56.8$ \\
$(19.8)$ \\
TE group $(n=143):$ \\
Age: \\
$\bullet 18<$ age $\leq 40(49.7 \%)$ \\
$\bullet 40<$ age $\leq 60(50.3 \%)$ \\
Gender: \\
$\bullet$ Male $(43.4 \%)$ \\
$\bullet$ Female $(56.6 \%)$ \\
VAS pain average $(0-100 \mathrm{~mm}): 57.7$ \\
$(19.9)$
\end{tabular}

Inclusion criteria: (1) LBP $\geq 12$ wk with or without pain radiating into the $\operatorname{leg}(\mathrm{s})$ and (2) age of 18-60 y.

Exclusion criteria: (1) symptoms of serious spinal pathology such as malignancy, osteoporosis, vertebral fracture, and spinal stenosis, (2) clinical symptoms of an acute herniated disk accompanied by nerve root entrapment, unstable spondylolisthesis, and spondylitis, (3) health conditions that prevented them from performing strenuous exercise, and (4) language problems.

Intervention Group A received a program of combined investigated exercise, education, and pain management. They were reassured that there was no serious cause for their back pain and that the exercise program was safe and effective.

12-wk program:

- Weeks 1-4: exercises completed 3 times per week for 2 -h sessions and biweekly lessons on anatomy, postural techniques, and pain management for $10 \mathrm{~h}$ in total.

-Weeks 5-8: 2-h exercise sessions twice a week at the study site and once at home.

- Weeks 9-12: 2-h exercise sessions 3 times a week at home.

Group B received a program of specific and intensive muscle training exercises to strengthen and shorten the muscles of the back and gluteus region for $1 \mathrm{~h}$ twice a week for $12 \mathrm{wk}$ with a physiotherapist.

\section{Bodes Pardo et al (2018) ${ }^{3}$}

Randomized controlled trial

65 patients recruited from 4 private physiotherapy practices and Alcala University were randomized into combined PE and TE group and TE-only group.

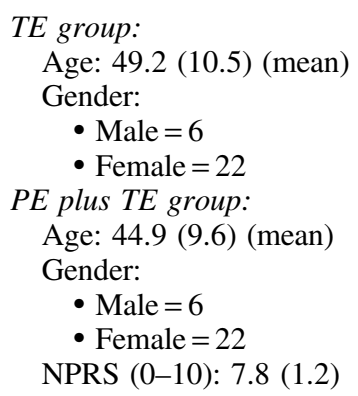

Inclusion criteria: (1) aged between 20 and $75 \mathrm{y},(2)$ experiencing chronic NS-LBP for $\geq 6 \mathrm{mo}$, and (3) Spanish as native language.

Exclusion criteria: (1) diagnosis of lumbar radiculopathy, (2) receiving treatment for their chronic NS-LBP at the time of the study, (3) pain related to tumors or infections, metastases, osteoporosis, inflammatory arthritis, or fractures, and (4) known mental disorders diagnosed by a physician.

All participants received a TE program that consisted of motor control exercise for the lumbar spine, stretching, and aerobic exercise.

The TE program was demonstrated and corrected to them during the first session, and then, those were completed at home everyday for a month, reexamined, and continued alone for 2 more months. The combined TE and PE group also received 2 education sessions lasting $30-50 \mathrm{~min}$ in small groups and a leaflet with the content.

The PE content consisted of verbal explanation and visual presentation of the neurophysiology of pain.

\section{Pires et al (2015)}

Randomized controlled trial

62 participants recruited from a waiting list of a Portuguese outpatient clinic were randomly assigned to groups with either aquatic therapy alone or combined aquatic therapy and pain neurophysiology.

Education group $(n=30)$ :

Age: 50.9 (6.2) (mean)

Gender:

- Male $=10$

- Female $=20$

Pain intensity (0-100): 43.4 (22.9)

(mean)

Control group $(n=32)$ :

Age: 51.0 (6.3) (mean)

Gender:

- Male $=12$

- Female $=20$

Pain intensity (0-100): 42.4 (21.2) (mean)

Inclusion criteria: (1) LBP $\geq 3$ mo, (2) aged between 18 and $65 \mathrm{y}$, and (3) literate in Portuguese.

Exclusion criteria: (1) clinical signs of an infection, tumor, osteoporosis, fracture, structural deformity, inflammatory disorder, radicular syndrome, or cauda equina syndrome, (2) history of back surgery or conservative treatment in the prior 6 and 3 mo, respectively, (3) pregnancy, and (4) cardiac and/or respiratory condition impediment to physical exercise.

All participants performed a 6-wk program consisting of 12 biweekly sessions of aquatic exercise based in a therapeutic pool. The PE program consisted of two 90-min sessions that addressed acute pain origin in the nervous system, transition from acute to chronic pain, central sensitization, the role of the brain in the perception of pain, psychosocial factors related to pain, cognitive and behavioral responses related to pain, flare-up management, and pacing. 
Table 2 (continued)

\begin{tabular}{lll}
\hline & Dufour et al (2010) & Bodes Pardo et al (2018) \\
\hline Outcome & Primary: & Primary: \\
measures & (1) VAS & (1) NPRS for NS-LBP \\
& Secondary: & Secondary: \\
& (1) RMDQ & (1) RMDQ \\
& (2) SF-36 & (2) PCS \\
& (3) Central Sensitization Inventory \\
& (4) TSK-11 measures at baseline and at $1-$ \\
& and 3-mo postintervention \\
& (5) PGIC at 1- and 3-mo postintervention \\
& (6) Pressure pain thresholds and physical \\
& performance (finger to nose tests) measured \\
& by blinded assessors at baseline and 1-mo \\
& follow-up
\end{tabular}

Main

findings

Level of

evidence

Validity

score

Conclusion
Primary outcome measures:

- Group A had a 38.7-mm decrease in VAS pain average at the 3-mo follow-up.

- Group B had a 45.7-mm decrease in

VAS pain average at the 3-mo follow-up.

- Neither group had a significant decrease in pain at the 3-mo follow-up $(P>.05)$.

- There was no statistical significance $(P=.23)$ between the groups.

- There was no significance $(P=.75)$ between analgesic medication intake of both groups.

Secondary outcome measures:

- Significant improvements in RMDQ occurred in both group A (24\%) and group B (12\%).

- Both group A and group B had a significant improvement $(P<.05)$ in 8 out of 10 dimensions of the SF- 36 .

2

N/A

Both groups showed long-term improvement in pain and disability scores with only minor differences in statistical results between groups.

Further research needs to be conducted to decide if one intervention is more effective than the other.

\section{Primary outcome measures:}

- The TE group had a 3.0-point average decrease at the 3 -mo follow-up $(P<.05)$.

- The PE plus TE group had a 5.3-point average decrease at the 3-mo follow-up $(P<.05)$.

- The PE plus TE group had a more statistically significant decrease in pain intensity compared with the TE group.

- The PE plus TE group had a significant difference in decreased pain intensity compared with the TE group $(P<.001)$. Secondary outcome measures:

- The PE plus TE group had a greater significant improvement $(P<.001)$ in RMDQ compared with the TE group.

- The PE plus TE group had a greater significant improvement $(P<.001)$ in lumbar pressure pain thresholds compared with the TE group.

- The PE plus TE group had a greater significant improvement $(P<.05)$ in fingerto-floor distance test compared with the TE group.

- The PE plus TE group had a greater significant improvement $(P<.05)$ in PGIC compared with the TE group.

2

N/A

Implementing a PE program combined with TE is found to be more effective in reducing pain, disability, and pain catastrophizing compared with solely therapeutic education in patients with CLBP.

This article can be used for further research on CLBP and effective interventions to decrease pain and disability. This study can only be used for short-term outcomes no longer than a time frame of 3 mo.

\section{Pires et al (2015) ${ }^{4}$}

Primary:

(1) VAS

(2) Quebec Back Pain Disability Scale Secondary:

(1) TSK-11 assessed at baseline, $6 \mathrm{wk}$, and $3 \mathrm{mo}$

Primary outcome measures:

- The education group had a 25.4-mm decrease in VAS pain average at the 3-mo follow-up $(P<.05)$.

- The control group had a 6.6-mm decrease in VAS pain average at the 3-mo follow-up $(P>.05)$.

- The education group had a significant decrease in pain intensity $(P<.05)$ compared with the control group at the 3-mo follow-up.

- There was no significant decrease $(P=.09)$ in functional disability for either group at the 3-mo follow-up.

- There was higher chance of the education group reporting benefits for pain intensity (58.6\%) and functional disability $(72.4 \%)$ from the intervention compared with the control group $(40.7 \%$ and $44.4 \%$, respectively).

Secondary outcome measure:

- There is no statistical correlation with pain intensity and functional disability affecting kinesiophobia.

2

N/A

Beginning treatment with PE, followed by aquatic therapy is clinically more effective in regard to the reduction of pain and functional disability than only implementing aquatic therapy for patients with CLBP. This study can be used for research on short-term outcomes within a time frame of 3 mo. Further research is necessary to determine a better understanding of how PE influences pain intensity and disability in long-term effects and outcomes past a time frame of 3 mo.

Abbreviations: LBP, low back pain; N/A, not applicable; NPRS, numerical pain rating scale; NS-LBP, nonspecific low back pain; PCS, pain catastrophizing scale; PE, pain neurophysiology education; PGIC, Patient Global Impression of Change; RMDQ, Roland-Morris Disability Questionnaire; SF-36, Short Form-36; TE, therapeutic exercise; TSK-11, Tampa Scale for Kinesiophobia; VAS, visual analog scale; CLBP, chronic low back pain.

disability and kinesiophobia have also been observed following this intervention. Pain education is a tool that can help clinicians to practice with a biopsychosocial and patient-centered model to give the best possible care.
While the purpose of this critically appraised topic was focused on evaluating change in patient-reported pain rating scales, other outcome measures were taken in these 3 studies that can be of clinical use for the rehabilitation clinician. Dufour et $\mathrm{al}^{2}$ observed a 
significant difference between the pain education and therapeutic exercise group, and the therapeutic exercise group alone in regards to the Roland-Morris Disability Questionnaire $(P=.003)$ as well as the MOS Short Form-36 Health Survey, specifically in the physical functional and component $(P<.001$ and $P=.001$, respectively) in favor of the combined pain education and therapeutic exercise group. The authors did not find any significant difference in Global Perceived Outcome or ability to work. Bodes Pardo et $\mathrm{al}^{3}$ observed significant differences in pain intensity $(P<.001)$, Roland-Morris Disability Questionnaire $(P<.001)$, Pain Catastrophizing Scale $(P<.001)$, Tampa Scale for Kinesiophobia $(P<.001)$, lumbar pressure pain threshold $(P<.001)$, and finger-to-floor distance $(P<.05)$ when pain education plus therapeutic exercise group was compared with therapeutic exercise alone group. Pires et $\mathrm{al}^{4}$ found significant differences in pain intensity at 3-month follow-up favoring the pain education plus therapeutic exercise group, but no significant differences were found for kinesiophobia or functional disability between the groups.

Dufour et $\mathrm{a}^{2}$ compared 2 different therapeutic exercise programs and did not find a significant difference between the 2 experimental groups in regards to patient-reported pain (Table 2). In their study, group A patients, who received pain education in addition to therapeutic exercise, gradually transitioned from physiotherapist-supervised exercise to home exercises over the 12 weeks. Group B, the therapeutic exercise only group, received supervised exercise over the entire 12 weeks. This detail could contribute to the nonsignificant finding in patient-reported pain due to uncontrolled variables, such as patient compliance and selfefficacy. Some limitations of the Bodes Pardo et $\mathrm{al}^{3}$ include a small sample size, no exclusion of selection bias, and what the participants did outside of the study was not recorded (other treatment, activity, etc). Limitations associated with the Pires et $\mathrm{al}^{4}$ study include a small sample size, no blinding of the physiotherapists conducting the therapeutic exercise and pain education, and there was no testing of the participants to record their understanding of pain education. A major limitation of the Dufour et $\mathrm{al}^{2}$ article has previously been discussed.

The results highlighted in this appraisal directly echo similar theories of chronic pain, such as the fear-avoidance model..$^{15,16}$ The fear-avoidance model theorizes a chronic pain cyclic effect of dysfunctional thought patterns and ideas of a patient living with chronic pain that keeps them from progressing in treatment despite the physical remission of previous pathology. ${ }^{15,16}$ Patients with LBP, regardless if it is specific or nonspecific pain, ${ }^{17}$ or if the patient in an athlete or nonathlete, ${ }^{8}$ have been associated with having elevated variables of the fear-avoidance model. Thus, including pain education along with therapeutic interventions is a necessary component of managing patients with chronic NS-LBP and may help reduce pain and increase function compared with therapeutic exercise alone.

Future research in this area should look at whether pain education reduces the duration of rehabilitation for chronic NSLBP as well as to investigate long-term versus short-term effects of this combination. Future research considerations should also focus on whether pain education along with therapeutic exercise is efficacious in athletic populations as athletes tend to have different motivations and stressors that could affect the results on these interventions. ${ }^{18}$ This CAT should be reviewed in 2 years or when additional best evidence has been published that may change the clinical bottom line for the research question posed in this review.

\section{References}

1. Trompeter K, Fett D, Platen P. Prevalence of back pain in sports: a systematic review of the literature. Sports Med. 2017;47(6):11831207. PubMed ID: 28035587 doi:10.1007/s40279-016-0645-3

2. Dufour N, Thamsborg G, Oefeldt A, Lundsgaard C, Stender S. Treatment of chronic low back pain: a randomized, clinical trial comparing group-based multidisciplinary biopsychosocial rehabilitation and intensive individual therapist-assisted back muscle strengthening exercises. Spine. 2010;35(5):469-476. PubMed ID: 20147878 doi:10.1097/BRS.0b013e3181b8db2e

3. Bodes Pardo G, Lluch Girbés E, Roussel NA, Gallego Izquierdo T, Jiménez Penick V, Pecos Martín D. Pain neurophysiology education and therapeutic exercise for patients with chronic low back pain: a single-blind randomized controlled trial. Arch Phys Med Rehabil. 2018;99(2):338-347. PubMed ID: 29138049 doi:10.1016/j.apmr. 2017.10.016

4. Pires D, Cruz EB, Caeiro C. Aquatic exercise and pain neurophysiology education versus aquatic exercise alone for patients with chronic low back pain: a randomized controlled trial. Clin Rehabil. 2015;29(6):538-547. PubMed ID: 25200879 doi:10.1177/ 0269215514549033

5. Heidari J, Belz J, Hasenbring M, Kleinert J, Levenig C, Kellmann M. Examining the presence of back pain in competitive athletes: a focus on stress and recovery. J Sport Rehabil. 2019;28(2):188-195. PubMed ID: 29140183 doi:10.1123/jsr.2017-0235

6. Chehadi O, Rusu AC, Konietzny K, et al. Brain structural alterations associated with dysfunctional cognitive control of pain in patients with low back pain. Eur J Pain. 2018;22(4):745-755. PubMed ID: 29239055 doi:10.1002/ejp.1159

7. Gajsar H, Titze C, Levenig C, et al. Psychological pain responses in athletes and non-athletes with low back pain: avoidance and endurance matter. Eur J Pain. 2019;23(9):1649-1662. PubMed ID: 31220382 doi:10.1002/ejp.1442

8. Hulsebusch J, Hasenbring MI, Rusu AC. Understanding pain and depression in back pain: the role of catastrophizing, help-/ hopelessness, and thought suppression as potential mediators. Int $J$ Behav Med. 2016;23(3):251-259. PubMed ID: 26590138 doi:10. 1007/s12529-015-9522-y

9. Alrwaily M, Timko M, Schneider M, et al. Treatment-based classification system for low back pain: revision and update. Phys Ther. 2016; 96(7):1057-1066. PubMed ID: 26637653 doi:10.2522/ptj.20150345

10. Dreisinger TE. Exercise in the management of chronic back pain. Ochsner J. 2014;14(1):101-107. PubMed ID: 24688341

11. Chou R, Turner JA, Devine EB, et al. The effectiveness and risks of long-term opioid therapy for chronic pain: a systematic review for a National Institutes of Health Pathways to Prevention Workshop. Ann Intern Med. 2015;162(4):276-286. PubMed ID: 25581257 doi:10. 7326/M14-2559

12. Moseley GL, Butler DS. Fifteen years of explaining pain: the past, present, and future. J Pain. 2015;16(9):807-813. PubMed ID: 26051220 doi:10.1016/j.jpain.2015.05.005

13. Cherkin DC, Sherman KJ, Balderson BH, et al. Effect of mindfulnessbased stress reduction vs cognitive behavioral therapy or usual care on back pain and functional limitations in adults with chronic low back pain: a randomized clinical trial. JAMA. 2016;315(12):1240-1249. PubMed ID: 27002445 doi:10.1001/jama.2016.2323

14. Lee SA, Edget DM. Cognitive Behavioral Therapy: Applications, Methods and Outcomes. Hauppauge, NY: Nova Science Publishers, Inc; 2012.

15. Crombez G, Eccleston C, Van Damme S, Vlaeyen JW, Karoly P. Fear-avoidance model of chronic pain: the next generation. Clin 
J Pain. 2012;28(6):475-483. PubMed ID: 22673479 doi:10.1097/ AJP.0b013e3182385392

16. Vlaeyen JW, Crombez G, Linton SJ. The fear-avoidance model of pain. Pain. 2016;157(8):1588-1589. PubMed ID: 27428892 doi:10. 1097/j.pain.0000000000000574

17. Lundberg M, Frennered K, Hagg O, Styf J. The impact of fearavoidance model variables on disability in patients with specific or nonspecific chronic low back pain. Spine. 2011;36(19):1547-1553. doi:10.1097/BRS.0b013e3181f61660

18. Forsdyke D, Smith A, Jones M, Gledhill A. Psychosocial factors associated with outcomes of sports injury rehabilitation in competitive athletes: a mixed studies systematic review. Br J Sports Med. 2016;50(9):537-544. PubMed ID: 26887414 doi:10.1136/bjsports2015-094850 